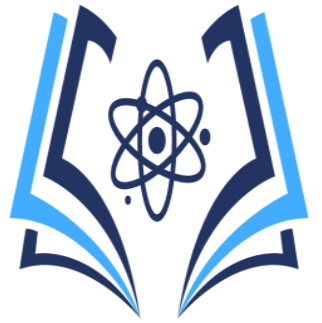

\title{
Infecciones de vías urinarias en mujeres, su conducta y
}

\section{factores de riesgo}

\section{Urinary tract infections in women, their behavior and risk factors \\ Infecciones de vías urinarias en mujeres}

\begin{abstract}
Dr. Roberth Zambrano Santos PhD
Universidad Estatal del Sur de Manabí - Instituto Superior Universitario Portoviejo, rzambranosantos@yahoo.es, Código Orcid: https://orcid.org/0000-0002-4072-4738.
\end{abstract}

Recibido: 29-09-2019
Contacto: rzambranosantos@yahoo.es

\section{Resumen}

El presente trabajo tuvo como origen la identificación de las conductas y factores de riesgo de las infecciones de vías urinaria (IVU) en mujeres, debido al alto porcentaje de pacientes con estas afectaciones, el objetivo fue: determinar las conductas y factores biológicos de IVU en mujeres, los objetivos específicos fueron: Analizar los factores sociodemográficos y biológicos que influyen en la aparición de IVU en mujeres; Identificar los tipos de infecciones de vías urinarias de mayor incidencia en mujeres. Explicar los signos y síntomas de las IVU en mujeres. La investigación fue cualitativa, de tipo descriptiva, por las condiciones propias de la pandemia la recopilación de la información fue por los medios digitales. En conclusión se determina que las infecciones urinarias siguen siendo una de las formas más comunes de infección tanto en la comunidad como, en particular, en el ámbito de la atención médica, convirtiéndose en una preocupación importante y causan una carga personal y social sustancial debida tanto a su prevalencia como a la capacidad de los microbios para compartir mecanismos de resistencia.

Palabras clave: Educación para la salud, enfermedades ginecológicas, infecciones en mujeres Abstract

The present work had as its origin the identification of the behaviors and risk factors of urinary tract infections (UTI) in women, due to the high percentage of patients with these affectations, the objective was: to determine the behaviors and biological factors of UTI in women, the specific objectives were: To analyze the sociodemographic and biological factors that influence the appearance of UTI in women; Identify the types of urinary tract infections with the highest incidence in women. Explain the signs and symptoms of UTIs in women. The research was qualitative, descriptive, due to the conditions of the pandemic, the collection of information was by digital media. In conclusion, it is determined that urinary tract infections continue to be one of the most common forms of infection both in the community and, in particular, in the field of medical care, becoming a major concern and causing a substantial personal and social burden due to both to its prevalence and to the ability of microbes to share resistance mechanisms.

Key words: Health education, gynecological diseases, infections in women

\section{Introducción}

Las infecciones del tracto urinario (ITU) son una de las enfermedades microbianas más comunes encontradas en la práctica médica que afectan a personas de todas las edades. En todo el mundo, la prevalencia de las infecciones urinarias se estimó en alrededor de 150 millones de personas por año. Las infecciones del tracto urinario generalmente se encuentran entre las infecciones bacterianas más comunes y son una queja frecuente de las mujeres que visitan a sus ginecólogos (1). El diagnóstico y el tratamiento precisos de las infecciones urinarias desempeñan un papel importante en la atención médica rentable y la utilización adecuada de antimicrobianos.

En Ecuador, la prevalencia en mujeres fue de 14,6\%. En Quito, se encontró que las infecciones urinarias eran de $(13,3 \%)$ y tenía una tasa de resistencia a los medicamentos del 20 al $60 \%$ entre las madres prenatales La resistencia a los antimicrobianos es un problema mundial, y se ha planteado la preocupación de que algunas infecciones para las que ahora se dispone de tratamiento puedan volverse intratables. El uso empírico generalizado de antibióticos, si bien es conveniente, contribuye potencialmente al desarrollo de resistencia a los antimicrobianos. Por lo tanto, la evolución de la resistencia a los antimicrobianos, requiere una reevaluación constante de la terapia antimicrobiana empírica (2).

La problemática de la infección del tracto urinario no es un diagnóstico definido por el laboratorio. El diagnóstico debe basarse en los síntomas clínicos siempre que sea posible confirmarse mediante microscopía y cultivo de orina positivos. No 
obstante, en Manabí en el distrito de salud reposan datos sobre la prevalencia de infecciones urinarias que fue de $67 / 300(22,33 \%)$ y Escherichia coli fue el uropatógeno bacteriano más prevalente con 41/67 $(61,19 \%)$ seguido de Staphylococcus aureus 10/67 $(14,93 \%)$.

Los ginecólogos con frecuencia evalúan a los pacientes en busca de infecciones urinarias. Algunas percepciones erróneas de la interpretación de los síntomas del paciente y los resultados de laboratorio conducen a un sobretratamiento de la IVU (3). En este caso se busca conocer la conducta de la infección, así como los factores biológicos que inciden en las infecciones recurrentes que son comunes y es importante que se controlen $y$ prevengan de manera efectiva.

Las infecciones urinarias son las infecciones ambulatorias más comunes en los Estados Unidos (EEUU). Con la excepción de un pico en mujeres jóvenes de 14 a 24 años, la prevalencia de infecciones urinarias aumenta con la edad. La prevalencia en mujeres mayores de 65 años es aproximadamente del $20 \%$, en comparación con aproximadamente el $11 \%$ en la población general. Entre el $50 \%$ y el $60 \%$ de las mujeres adultas tendrán al menos una IVU en su vida, y cerca del $10 \%$ de las mujeres posmenopáusicas indican que tuvieron una IV en el año anterior (5).

La infección de vías urinarias (IVU) es una de las consultas por concepto infeccioso más frecuente del servicio de urgencias, principalmente en los pacientes adultos mayores que ha generado entre 7 millones y 8.4 millones de consultas a los servicios de urgencias en países desarrollados. Se ha visto con preocupación la variación epidemiológica de la IVU, encontrando un incremento en el número de casos secundarios a microorganismos productores de betalactamasas de espectro extendido (BLEE), con las implicaciones sociales, económicas y morbilidad que esta acompaña (6)

Los síntomas del tracto urinario en mujeres se caracterizan por la urgencia de orinar, la incontinencia diurna, las maniobras de retención y un aumento o disminución de la frecuencia de la micción en ausencia de cualquier enfermedad neurológica o anomalía anatómica del tracto urinario inferior. Las beta-lactamasas de espectro extendido son un grupo de beta-lactamasas que comparten la habilidad de hidrolizar las cefalosporinas de tercera generación y el aztreonam pero son inhibidas por el ácido clavulánico (7).

Los factores biológicos más comunes de infección pueden ser bacterias u hongos. Los factores de riesgo incluyen la anatomía femenina, las relaciones sexuales, la diabetes, la obesidad y los antecedentes familiares. Aunque las relaciones sexuales son un factor de riesgo, las IVU no se clasifican como infecciones de transmisión sexual (ITS). Entre los
Vol. 1, Nro. 1, Publicado 2019-12-31

factores no comunes se encuentra la vejiga hiperactiva es el trastorno del tracto urinario inferior más común; sin embargo, queda por aclarar su asociación con IVU (8).

En un estudio también se demostró que la micción infrecuente es un factor de riesgo febril tanto en el análisis univariado como en el multivariado, y puede considerarse un predictor independiente. Este síntoma podría conducir al desarrollo de una infección urinaria al facilitar la acumulación de orina residual, un factor de riesgo conocido de infección urinaria. Las niñas tienen más probabilidades de posponer la micción en comparación con los niños y esto puede explicar el hallazgo del sexo como un predictor independiente de IVU febril; sin embargo, el aplazamiento de la evacuación como factor de riesgo no alcanzó significación estadística (9).

Por lo anteriormente mencionado a través de este estudio de revisión bibliográfica, se pretende determinar las infecciones más comunes así como conductas y factores de riesgos más frecuentes que causan IVU en la mujer.

\section{Materiales y métodos}

Se realizó un estudio bibliográfico, que se trata de un estudio basado en una serie de resúmenes de publicaciones de fuentes científicas de revistas digitales. Los artículos generales se utilizaron para tomar datos relevantes al tema y extraer una estructura lógica de contenidos. Se tomó referencias de las revistas tales como: Scielo, PLOS - Public Library of Science, Revues.org, Portal de Revistas Científicas Complutenses, Ciencia, Portal de revistas digitales de la UPV/EHU, E-Revistas publicadas por las Universidades Españolas, REDIB, Free Medical Journals.

En la búsqueda se tomaron 26 documentos de información realizada a partir del año 2006 a 2019.

\section{Criterios de inclusión}

Documentos bibliográficos relacionados en el tema a investigar

Para el desarrollo del análisis bibliográfico, se realizó una selección de artículos científicos, incluyendo la indagación de las bases de datos de revistas científicas digitales tales como: Scielo, PLOS - Public Library of Science, Revues.org, Portal de Revistas Científicas Complutenses, Ciencia, Portal de revistas digitales de la UPV/EHU, E-Revistas publicadas por las Universidades Españolas, REDIB, Free Medical Journals.

\section{Criterios de exclusión}

Artículos científicos que no se encuentren relacionados con la temática.

Los métodos utilizados fueron los empíricos (observacional) y teóricos (analítico y sintético), porque se basan en el análisis y determinación de resultados a partir de teorías relevantes. Se utilizó la técnica de la observación. 


\section{Resultados}

En la investigación se buscó determinar las conductas y factores biológicos de IVU en mujeres para comprender los factores de riesgo individuales y específicos de la población asociados con las infecciones recurrentes del tracto urinario (IVU), los antecedentes de este estudio han permitido establecer los factores comprobados de las infecciones.

En el objetivo específico 1, se analizaron los factores sociodemográficos y biológicos que influyen en la aparición de IVU en mujeres.

En análisis del contenido, las infecciones del tracto urinario inferior (IVU) se encuentran entre las infecciones bacterianas más frecuentes y representan una morbilidad considerable entre las mujeres sexualmente activas. la mayoría de los factores de riesgo de infección inicial son potencialmente modificables, pero suficientes para presentar también riesgo de recurrencia. Muchos de los hallazgos reflejan las prácticas culturales y étnicas de nuestro país.

No existe una explicación definitiva de por qué solo algunas mujeres contraen infecciones urinarias y por qué las recurrencias tienden a ocurrir solo en algunos sujetos. Las infecciones repetidas generalmente ocurren en ausencia de anomalías anatómicas en el tracto urinario. Se sospecha que las características genéticas, conductuales y de virulencia bacteriana del hospedador influyen en la causalidad. Los factores del hospedador que pueden influir en el riesgo de infección urinaria incluyen: antecedentes sexuales, uso de anticonceptivos de barrera con espermicidas, retraso en la micción poscoital.

Se ha demostrado que la modificación del comportamiento reduce el riesgo de infección urinaria. Se encontró que las mujeres con infección urinaria recurrente tenían antecedentes familiares más fuertes de infección urinaria. La mayoría de estos estudios se han realizado en países desarrollados donde el nivel socioeconómico es más alto y muchas prácticas de comportamiento entre las mujeres difieren de las de los países en desarrollo.

Muchos de los factores de riesgo descritos para la población occidental difieren de la población de esta región; por ejemplo, el uso de espermicidas, jacuzzis y tampones. Hemos preguntado a todos los participantes sobre el jugo de arándano, ninguna de las mujeres respondió afirmativamente. $\mathrm{La}$ referencia dada es de nuestro estudio anterior, donde informamos un resultado similar. 
Tabla 1. Factores de riesgo para desarrollo de IVU

\begin{tabular}{|c|c|c|c|c|}
\hline \multicolumn{5}{|c|}{ Factores de riesgo para desarrollo de IVU } \\
\hline $\begin{array}{l}\text { Factores de } \\
\text { riesgo }\end{array}$ & Casos $n=183$ & $\begin{array}{l}\text { Controles } n= \\
183\end{array}$ & OR (IC 95\%) & Valor de $p$ \\
\hline $\begin{array}{l}\text { Hospitalización } \\
\text { previa, n. (\%) }\end{array}$ & $74(40,44)$ & $53(28,96)$ & $\begin{array}{l}1,66 \\
(1,05-2,63)\end{array}$ & 0,02 \\
\hline $\begin{array}{l}\text { Residente en } \\
\text { Unidad } \\
\text { Crónica, n. (\%) }\end{array}$ & $5(2,73)$ & $4(2,18)$ & $\begin{array}{l}1,25 \\
(0,26-6,43)\end{array}$ & 0,73 \\
\hline $\begin{array}{l}\text { Uso previo de } \\
\text { antibióticos, n. } \\
(\%)\end{array}$ & $101(55,19)$ & $66(36,06)$ & $\begin{array}{l}2,18 \\
(1,40-3,39)\end{array}$ & 0,0002 \\
\hline $\begin{array}{l}\text { Uso previo de } \\
\text { Corticoides, n. } \\
(\%)\end{array}$ & $10(5,46)$ & $7(3,82)$ & $\begin{array}{l}1,45 \\
(0,48-4,60)\end{array}$ & 0,45 \\
\hline $\begin{array}{l}\text { Instrumentación } \\
\text { del Tracto } \\
\text { Urinario, n. }(\%)\end{array}$ & $51(27,86)$ & $18(9,83)$ & $\begin{array}{l}3,54 \\
(1,91-6,74)\end{array}$ & $<0,0001$ \\
\hline $\begin{array}{l}\text { Uso de sonda } \\
\text { vesical, n. }(\%)\end{array}$ & $53(28,96)$ & $45(24,59)$ & $\begin{array}{l}1,25 \\
(0,76-2,04)\end{array}$ & 0,34 \\
\hline & & & & \\
\hline
\end{tabular}


Tabla 2. Factores de riesgo de infección del tracto urinario bacteriana

\begin{tabular}{|c|c|}
\hline \multicolumn{2}{|c|}{$\begin{array}{l}\text { Factores de riesgo de infección del tracto urinario bacteriana, pielonefritis y } \\
\text { candiduria en el trasplante renal }\end{array}$} \\
\hline Infección del tracto urinario bacteriana & $\begin{array}{ll}\text { - } & \text { Sexo femenino } \\
\text { - } & \text { Edad (por cada año) } \\
\text { - } & \text { Reflujo previo al trasplante } \\
\text { - } & \text { Donante fallecido } \\
\text { - } & \text { Tiempo con sonda vesical } \\
\text { - } & \text { Duración de la hospitalización } \\
\text { - } & \text { Incremento de inmunosupresión }\end{array}$ \\
\hline Pielonefritis aguda & $\begin{array}{ll}\text { - } & \text { Sexo femenino } \\
\text { - } & \text { Episodios de rechazo agudo } \\
\text { - } & \text { Número de infecciones del tracto } \\
\text { urinario } \\
\text { - }\end{array}$ \\
\hline Infección del tracto urinario por Candida & $\begin{array}{ll}\text { - } & \text { Sexo femenino } \\
\text { - } & \text { Ingreso en Unidad de Cuidados } \\
\text { - } & \text { Intensivos } \\
\text { - } & \text { Administración de antibióticos } \\
\text { - } & \text { Vonda vesical permanente } \\
\text { - } & \text { Malnutrición }\end{array}$ \\
\hline
\end{tabular}

Fuente: (18) 
Las infecciones del tracto urinario (ITU) son causadas por una amplia gama de patógenos, incluidas las bacterias Gram negativas y Gram positivas, así como los hongos. Las infecciones urinarias no complicadas suelen afectar a mujeres, niños y pacientes ancianos que por lo demás están sanos. Las infecciones urinarias complicadas generalmente se asocian con catéteres permanentes, anomalías del tracto urinario, inmunosupresión o exposición a antibióticos. El agente causal más común de las infecciones urinarias complicadas y no complicadas es la Escherichia coli uropatógena (UPEC). Para las infecciones urinarias no complicadas, otros agentes causales son Klebsiella pneumoniae, Staphylococcus saprophyticus, Enterococcus faecalis, Estreptococos del grupo B (GBS), Proteus mirabilis, Pseudomonas aeruginosa, Staphylococcus aureus y Candida spp. Para las infecciones urinarias complicadas, los otros agentes causales son Enterococcus spp., K. pneumoniae, Candida spp., S. aureus, P. mirabilis, P. aeruginosa y GBS (23).
ISSN 2773-7705

Periodo. Julio - Diciembre 2019

Vol. 1, Nro. 1, Publicado 2019-12-31

Las infecciones del tracto urinario (IVU) comienzan cuando los uropatógenos que residen en el intestino contaminan el área periuretral y pueden colonizar la uretra. La migración posterior a la vejiga y la expresión de pili y adhesinas da como resultado la colonización e invasión de las células paraguas superficiales. Las respuestas inflamatorias del huésped, incluida la infiltración de neutrófilos, comienzan a eliminar las bacterias extracelulares. Algunas bacterias evaden el sistema inmunológico, ya sea a través de la invasión de la célula huésped o mediante cambios morfológicos que resultan en resistencia a los neutrófilos, las bacterias se multiplican, y se forman biopelículas. Estas bacterias producen toxinas y proteasas que inducen daño a la célula huésped, liberando nutrientes esenciales que promueven la supervivencia bacteriana y la ascensión a los riñones (23).

En el objetivo específico dos, se identificaron los tipos de infecciones de vías urinarias de mayor incidencia en mujeres.

Tabla 3. Agentes infecciosos en IVU Patógeno

\begin{tabular}{|l|c|c|}
\hline Prevalencia (\%) & Complicada & tracto urinario \\
\hline UPEC & 75 & 75 \\
\hline K. neumonía & 6 & 8 \\
\hline S. saprophyticus & 6 & - \\
\hline Enterocuccus spp. & 5 & 11 \\
\hline Estreptococo del grupo B & 3 & 2 \\
\hline P. mirabilis & 2 & 2 \\
\hline P. aeruginosa & 1 & 2 \\
\hline S. aureus & 1 & 3 \\
\hline Candida spp. & 1 & 7 \\
\hline
\end{tabular}

Una infección del tracto urinario puede afectar diferentes secciones del tracto urinario, incluidas las siguientes (8):

Uretritis: infección de la uretra, el tubo hueco que drena la orina desde la vejiga hacia el exterior del cuerpo.

Cistitis: una infección bacteriana en la vejiga que a menudo se ha movido hacia arriba desde la uretra.

Pielonefritis: infección de los riñones que suele ser el resultado de una infección que se ha extendido por el tracto urinario o de una obstrucción en el tracto urinario. Una obstrucción en el tracto urinario hace que la orina regrese a los uréteres y riñones. En el objetivo específico 3, se explicaron los signos y síntomas de las IVU en mujeres.

Los síntomas comunes de una IVU son disuria, frecuencia urinaria, urgencia, dolor suprapúbico y posible hematuria. Los síntomas sistémicos suelen ser leves o ausentes. La orina puede tener un olor desagradable y parecer turbia. Las infecciones urinarias recurrentes son infecciones urinarias sintomáticas que siguen a la resolución de un episodio anterior, generalmente después del tratamiento adecuado. Son comunes entre las mujeres jóvenes y sanas, aunque estas mujeres generalmente tienen tractos urinarios anatómica y fisiológicamente normales (24).

Los síntomas de una IVU pueden incluir (24): 
- Una sensación de ardor al orinar

- Una necesidad frecuente o intensa de orinar, aunque poco sale cuando lo hace

- Orina turbia, oscura, con sangre o con olor extraño

- Sentirse cansado o tembloroso

- Fiebre o escalofríos

- Dolor o presión en la espalda o en la parte inferior del abdomen.

\section{Conclusiones}

- En conclusión, las infecciones urinarias siguen siendo una de las formas más
Periodo. Julio - Diciembre 2019

Vol. 1, Nro. 1, Publicado 2019-12-31

comunes de infección tanto en la comunidad como, en particular, en el ámbito de la atención médica.

- Son una preocupación importante y causan una carga personal y social sustancial debida tanto a su prevalencia como a la capacidad de los microbios para compartir mecanismos de resistencia.

- La carga de las infecciones urinarias tanto en las personas como en la sociedad es multifactorial y es probable que aumente en el contexto de la resistencia a los antibióticos.

\section{Colaboradores de la Investigación:}

Alcívar Vélez María Laura; Arteaga Cuadros Grachy Thays; Guillen Zambrano Mellanni Lisbeth; Loor Bravo Gema Luisa; Macías Rodríguez Emily Monserrate; Moran Vera Geomara Lisseth; Moreira Parrales Christel Gema.

\section{Bibliografía}

1. Diaz-Granados LES, Mendoza OES, Nuñez JFG. Características y factores de riesgo de la infección de vías urinarias. Infectio. $2018 ;:$ p. vol.22 no.3.

2. Toscano DER. Prevalencia de las Infecciones Genitourinarias en mujeres de 14 a 49 años. Universidad san francisco de quito. $2013 ; 1(1)$.

3. Gondim R, Azevedo R, Veiga ML. Factores de riesgo de infección del tracto urinario en niños con urgencia urinaria. Internacional braz j urol. 2018 Abr; 44(22).

4. Medina M, Castillo-Pino E. Introducción a la epidemiología y la carga de las infecciones del tracto urinario. Ther Adv Urol. 2019 Mayo; 1(Doi. 10.1177 / 1756287219832172).

5. Oscar Storme JTS, García-Mora A, Dehesa-Dávila M, Naber KG. Factores de riesgo y condiciones que predisponen a la infección del tracto urinario. Ther Adv Urol. 2019 Dic;(Doi: 10.1177 / 1756287218814382).

6. Minardi D, d'Anzeo G, Cantoro D, Conti A. Urinary tract infections in women: etiology and treatment options. Int J Gen Med. 2016 Abr;(Doi: 10.2147/IJGM.S11767).

7. Kabugo D, Kizito S, Ashok DD, Graham KA, Nabimba R. Factors associated with community-acquired urinary tract infections among adults attending. Afr Health Sci. 2016 Dic; 16(4).

8. Mishra B, Srivastava R, Agarwal J, Srivastava S, Pandey A. Factores de riesgo conductuales y psicosociales asociados con la cistitis primera y recurrente en mujeres indias. Indian J Community Med. 2016 Mar; 41(1).

9. Flores-Mireles AL, Walker JN, Caparon M, Hultgren SJ. Infecciones del tracto urinario: epidemiología, mecanismos de infección y opciones de tratamiento. Nat Rev Microbiol. 2016 Abr; 1(Doi: 10.1038 / nrmicro3432).

10 Inapanta F. Mayoclinc. [Online].; 2019 [cited 202009 1. Available from: https://www.mayoclinic.org/eses/diseases-conditions/urinary-tract-infection/symptoms- causes/syc-20353447.

11 Salvador R. Centro para prevencion de Enfermedades. [Online].; 2020 [cited 2020091.

. Available from: https://www.cdc.gov/antibiotic-use/community/sp/for-patients/common- illnesses/uti.html.

12 Guinzberg A, Arredondo, Amábile, Pacheco. Infecciones de Vias Urinarias (IVU).

. [Online].; 2015. Available from: 
https://cmu.org.mx/media/cms_page_media/57/GUIAS_MAPPA_IVU.pdf.

13 González MGM. Infección en las vias Urinarias. [Online].; 2018 [cited 2020091.

. Available from: https://nietoeditores.com.mx/nieto/suplemento_ivu.pdf.

14 Jiménez MA. Infeccion Urinaria. [Online].; 2018 [cited 202009 1. Available from:

. https://www.segg.es/tratadogeriatria/PDF/S35-05\%2042_III.pdf.

15 Mallén PD. Infecciones Urinarias. [Online].; 2019 [cited 202009 1. Available from:

. https://www.nefrologiaaldia.org/es-articulo-infecciones-urinarias-255.

16 Palma jc. Factores de riesgo que influyen en las mujeres con infeccion urinaria. Primera

. ed. ROSA MCL, editor. LIBERTAD ; 2013.

17 Palacio V. semfy. [Online].; 2019 [cited 202009 1. Available from:

. https://www.semfyc.es/wp-content/uploads/2016/07/8.1.-Infecci\%C3\%B3n-urinaria-en- la-mujer.pdf.

18 Pigrau C. Infecciones en el tracto Urinario. Salvat y caliidad. 2019 Mayo; 11(2).

.MARIELA TGA. Repositorio.upse. [Online].; 2013. Available from:

. https://repositorio.upse.edu.ec/bitstream/46000/1003/1/TESIS\%20INFECCIONES\%20\% 20URINARIAS.pdf.

19 Centro para el control y prevencion de enfermedades. [Online].; 2020. Available from:

. https://www.cdc.gov/antibiotic-use/community/sp/for-patients/common-illnesses/uti.html.

20 Velazquez E. slideshare. [Online].; 2016 [cited 202009 1. Available from:

. https://www.slideshare.net/eddynoy/infecciones-del-tracto-urinario-69429401.

21 Mariela TGA. Tesis Infeccion Urinaria. [Online].; 2013 [cited 202009 1. Available from:

. https://repositorio.upse.edu.ec/bitstream/46000/1003/1/TESIS\%20INFECCIONES\%20\% 20URINARIAS.pdf.

22 Serrallach CP. Servicio de Enfermedades Infecciosas. 2018; 12(2).

.Tan CW. Infecciones del tracto urinario en adultos. Singapur Med J. 2016 Sept; 57(9).

.Molin C, Valle ED, González L, Figueredo L. Infecciones urinarias en niños con vejiga

. neurogénica y los patrones de resistencia a los uropatógenos más frecuentes. Memorias del Instituto de Investigaciones en Ciencias de la Salud. 2018 Dic; 16(3).

23 Vigoa DAV. Revista cubana de Medicina. 2006 Agosto ; 34(2). 